\title{
qPCR-based methods for expression analysis of miRNAs
}

\author{
Diego A Forero ${ }^{\star 1,2}$, Yeimy González-Giraldo ${ }^{3}$, Luis J Castro-Vega ${ }^{4,5}$ \& George E Barreto ${ }^{3}$
}

\begin{abstract}
Several approaches for miRNA expression analysis have been developed in recent years. In this article, we provide an updated and comprehensive review of available qPCR-based methods for miRNA expression analysis and discuss their advantages and disadvantages. Existing techniques involve the use of stem-loop reverse transcriptionPCR, polyadenylation of RNAs, ligation of adapters or RT with complex primers, using universal or miRNA-specific qPCR primers and/ or probes. Many of these methods are oriented towards the expression analysis of mature miRNAs and few are designed for the study of pre-miRNAs and pri-miRNAs. We also discuss findings from articles that compare results from existing methods. Finally, we suggest key points for the improvement of available techniques and for the future development of additional methods.
\end{abstract}

\section{KEYWORDS \\ expression analysis - microRNAs - molecular assays • non-coding RNAs - real-time PCR}

'Laboratory of NeuroPsychiatric Genetics, Biomedical Sciences Research Group, School of Medicine, Universidad Antonio Nariño, Bogotá, Colombia; ${ }^{2} P h D$ Program in Health Sciences, School of Medicine, Universidad Antonio Nariño, Bogotá, Colombia; ${ }^{3}$ Departamento de Nutrición y Bioquímica, Pontificia Universidad Javeriana, Bogotá, Colombia; ' INSERM, UMR970, Paris-Cardiovascular Research Center, Equipe Labellisée par la Ligue contre le Cancer, Paris, France; ${ }^{5}$ Université Paris Descartes, Sorbonne Paris Cité, Faculté de Médecine, Paris, France; *Author for correspondence: diego.forero@ uan.edu.co

BioTechniques 67: 192-199 (October 2019) 10.2144/btn-2019-0065

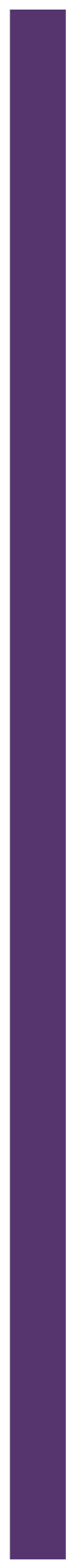

miRNA genes are a novel category of noncoding RNAs that have been involved in recent years in a large number of biological processes and in the pathophysiology of human diseases [1,2]. There are two main categories of miRNA genes, depending on their genomic localization: intronic and intergenic miRNAs [1]. In animals, primary miRNAs (pri-miRNAs) are commonly transcribed by RNA polymerase II and usually have a length of several kilobases. Pri-miRNAs are processed by the microprocessor complex to generate the precursor miRNAs (pre-miRNAs), which commonly have a length of around 70 nucleotides and are exported to the cytoplasm [1]. Pre-miRNAs are cleaved by Dicer into mature miRNAs, which are approximately 18-24 nucleotides long and are incorporated into the RNA-induced silencing complex in order to regulate the expression of target proteincoding genes. A short region in the mature miRNAs, called the 'seed', is the most important for binding to the 3 ' untranslated region of target genes [1]. The mechanisms of miRNA genesis and regulation in plants present some differences, in comparison with animals $[3,4]$.

The latest release of miRBase (version 22) [5] provides data for 38,589 pre-miRNAs from 271 organisms, including information for 1917 precursors and 2654 mature miRNAs in humans. As examples of other model organisms, it includes data for 326 hairpins and 428 mature sequences for Arabidopsis thaliana and 258 hairpins and 469 mature sequences for Drosophila melanogaster [5].

Considering the growing importance of miRNAs in multiple biological mechanisms, several methods based on qPCR for miRNA expression analysis have been developed in recent years [6-8]. In comparison to the traditional qPCR-based analysis of mRNA expression for protein-coding genes, the study of miRNA expression levels has particular features and challenges. In this article, we provide an updated and comprehensive review of available qPCRbased methods for miRNA expression analysis and discuss their advantages and disadvantages.

A search in PubMed was carried out to identify original articles that described qPCR-based methods for the analysis of miRNA expression [9]. Reference lists from review articles were also searched to identify additional primary publications of relevance.

Due to the short length of mature miRNAs and the high similarity of multiple members of miRNA families, expression analysis of these noncoding RNAs by qPCR has several particular challenges. Many available methods have taken advantage of the reverse transcription (RT) step, in multiple ways, in order to incorporate additional sequences that facilitate the amplification by PCR and its identification [10-14]. Available approaches use miRNA-specific or universal qPCR primers and an important number of techniques are based on the use of the SYBR Green dye, which has the advantage of being cheaper than the use of fluorescent probes, although probes provide additional specificity [15]. Due to multiple reasons, the largest number of techniques have been created for the analysis of mature miRNAs. In general, the methods with the largest number of citations correspond to the approaches described in the oldest articles.

As discussed in detail below, available methods have different disadvantages. The high similarity of members of miRNA subfamilies leads to problems of specificity with several techniques [16]. As many methods are focused on the analysis of mature miRNAs, in certain cases (in miRNAs with close paralogs) it is difficult to establish the genomic location of the dysregulated candidate. In other instances, the complex designs of some methods make their implementation in a standard molecular biology laboratory challenging. For some 


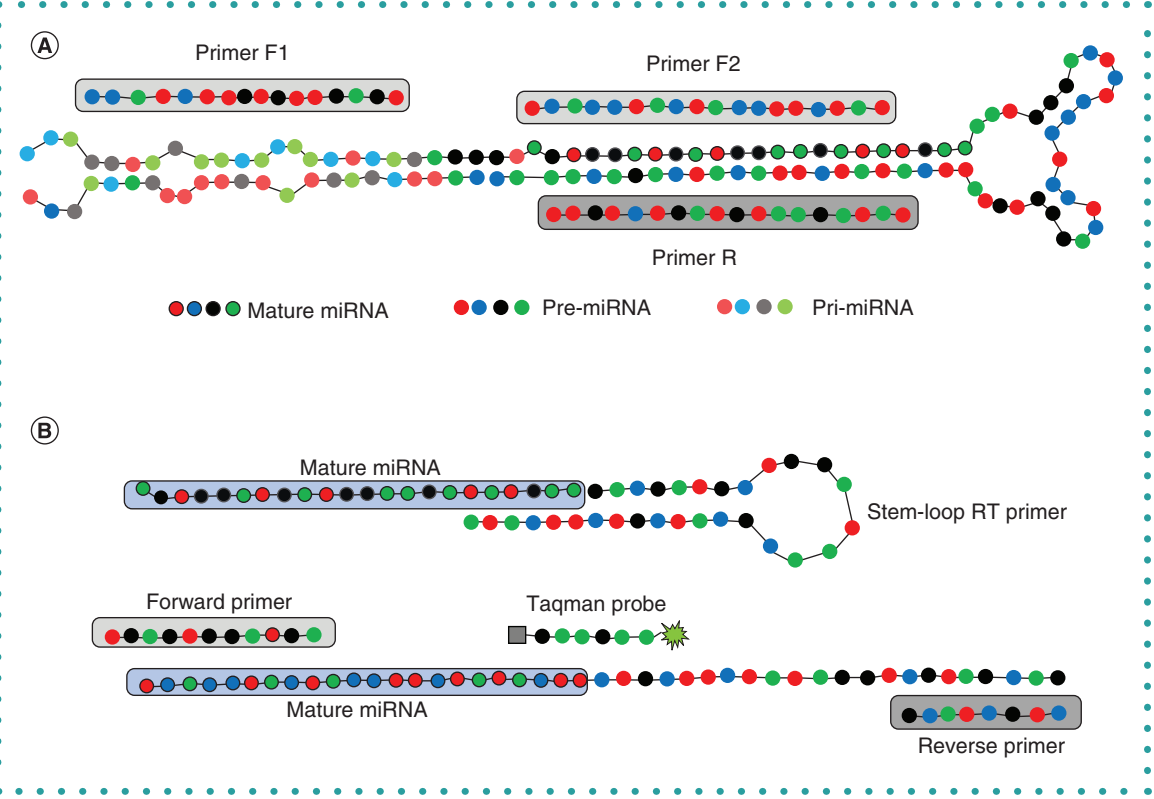

Figure 1. Overview of the qPCR methods developed by (A) Schmittgen et al. [10] and (B) Chen et al. [11]. The approach developed by Schmittgen et al. uses three primers that amplify pri- and pre-miRNA molecules and the method created by Chen et al. involves a stem-loop RT primer and a miRNA-specific TaqMan probe.

RT: Reverse transcriptase.

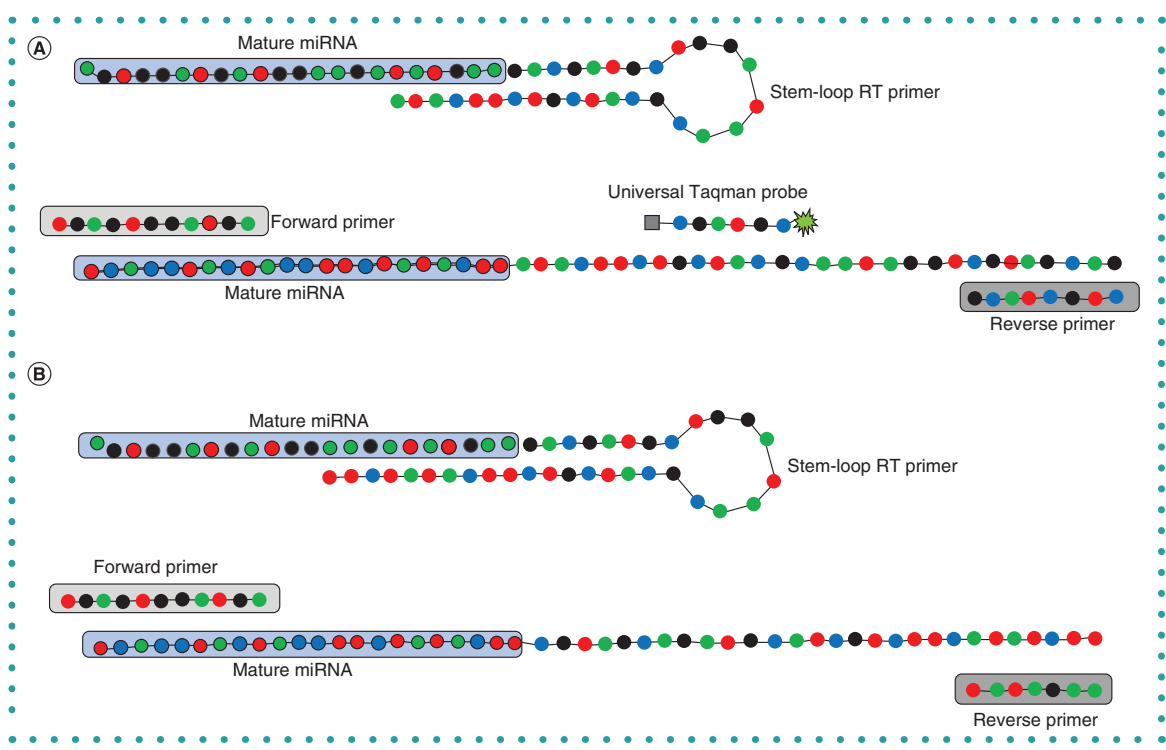

Figure 2. Overview of the qPCR methods developed by (A) Jung $U$ et al. [18] and (B) Tong et al. [19]. The approach developed by Jung et al. uses a stem-loop RT primer and a miRNA-universal TaqMan probe and the method created by Tong et al. involves a longer stem-loop RT primer and SYBR Green. RT: Reverse transcriptase.

$>$ techniques, the possibilities for costeffective multiplexing are limited.

\section{qPCR-BASED METHODS FOR THE ANALYSIS OF PRE- \& PRI-miRNAS}

In 2004, Schmittgen et al. reported the first qPCR-based method for the analysis of miRNAs [10]. This paper described the use included in both pri- and pre-miRNA molecules, and fluorescence from SYBR green is quantified.

\section{qPCR-BASED METHODS FOR THE ANALYSIS OF MATURE miRNAS}

\section{Approaches based on stem-loop RT}

In 2005, Chen et al. described the first use of a stem-loop qPCR approach for the analysis of mature miRNAs [11]. This method has been broadly used and it employs a stem-loop RT primer that binds to a mature miRNA (Figure 1B). The resulting cDNA is PCR-amplified with a miRNA-specific forward primer and a universal reverse primer; a miRNA-specific TaqMan probe is used and the fluorescence is measured to quantify mature miRNA levels [11]. Another group included a pre-amplification step to modify this protocol in order to allow multiplexing [17], and another laboratory modified this method to allow the use of a universal TaqMan probe (Figure 2A) [18]. A longer binding region for the miRNA sequence in the stem-loop RT primer (11 bp instead of $6 \mathrm{bp}$ ) led to a higher specificity and demonstrated that use of SYBR Green is a costeffective approach with this method (Figure 2B) [19]. It has been found that different stem-loop RT primers allow specific identifications of closely related members of a miRNA family (let-7) [16] .

\section{Approaches based on polyadenylation}

Shi and Chiang used the poly(A) polymerase to polyadenylate mature miRNAs and employed a poly $(\mathrm{T})$ adapter to generate a cDNA [12]. A miRNA-specific forward primer and a reverse primer that binds a region in the poly(T) adapter are used for PCR amplification, whereby fluorescence from SYBR green is measured (Figure $3 \mathrm{~A}$ ). A related method was developed, which required polyadenylation of mature miRNAs, use of a poly $(T)$ adapter to generate $\mathrm{CDNA}$, and a miRNA-specific forward primer and a universal reverse primer for PCR amplification [20].

Another similar approach was created, which is based on the polyadenylation of mature miRNAs and use of a poly $(T)$ adapter to generate CDNA, but it uses two miRNA-specific forward and reverse PCR primers and quantification with SYBR green (Figure 3B) [21]. Another work reported an 
approach that is based on polyadenylation of RNA, the use of an oligonucleotide (with a polyT region) that serves for $R T$ and that has binding sites for a universal reverse PCR primer and for a universal TaqMan probe. PCR is carried out with a miRNA-specific forward primer and a universal reverse PCR primer and fluorescence from the TaqMan probe is detected (Figure 4A) [22].

\section{Approaches based on ligation}

One of the methods uses a ligase for circularization of miRNAs, RT of the circularized miRNA and qPCR with overlapping primers and SYBR Green [24]. Moreover, another approach was created that is based on the ligation of a universal DNA adaptor to the mature miRNAs, the use of a universal RT primer that has a binding site for a universal reverse primer. qPCR is carried out with a miRNA-specific forward primer, a universal reverse primer and SYBR Green [25].

The miQPCR method involves the ligation of an oligonucleotide that includes binding regions for a RT primer and for a universal reverse PCR primer; PCR is carried out with a miRNA-specific forward primer, a universal reverse primer and SYBR Green (Figure 4B) [23].

It has been shown that the original stem-loop method developed by Chen et al. [11] failed to specifically identify $5^{\prime}$ and $3^{\prime}$ variants of miRNAs, resulting in the development ofthe Dumbbell-PCR method, which involves the ligation of a stem-loop oligonucleotide that binds specifically to a mature miRNA isoform. It uses a miRNA-specific RT primer and a universal forward primer and a miRNA-specific reverse primer. An miRNAspecific TaqMan probe is employed and the resulting fluorescence is detected [26].

\section{Other approaches}

Another method was developed, which is based on the use of a linear miRNA-specific RT primer, a miRNA-specific forward primer for overlap PCR, a universal forward primer and a miRNA-specific primer for qPCR. A miRNA-specific TaqMan probe is used and the fluorescence is employed to quantify mature miRNA levels [27]. Another method comprises the binding of a mature miRNA to a probe that has two hairpins in the extremes, cleavage by restriction enzymes, and the use of bridging oligos; PCR is carried out with two biotinylated primers and

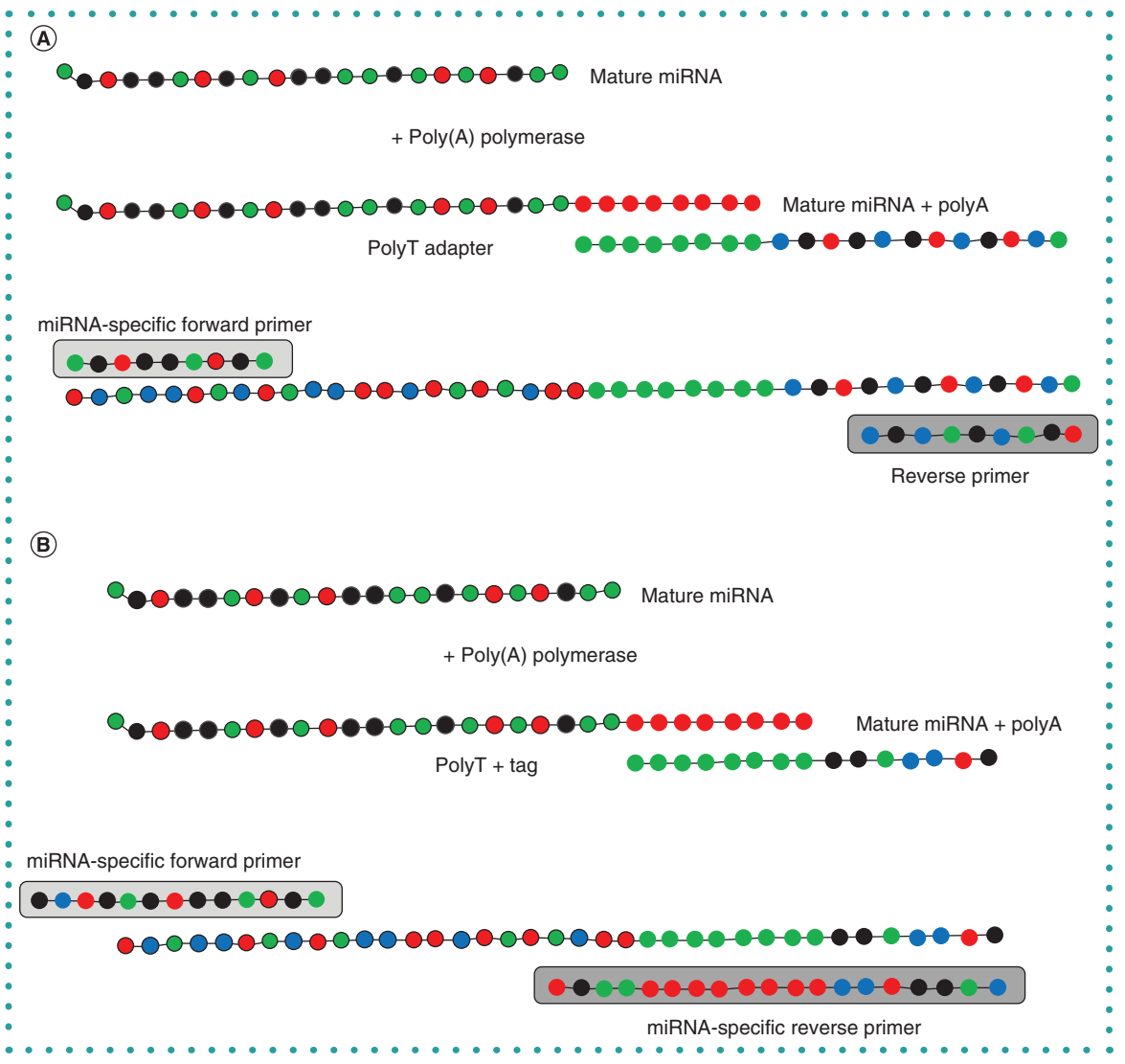

Figure 3. Overview of the qPCR methods developed by (A) Shi et al. [12] and (B) Balcells et al. [21]. The approach developed by Shi et al. uses polyadenylation, a polyT adapter and a miRNA-specific forward primer. The method created by Balcells I et al. also involves polyadenylation and a polyT adapter but employs two miRNA-specific primers.

colorimetry is employed [28].

An article reported an approach that carries out RT with a pincer probe and $\mathrm{QPCR}$ is done with miRNA-specific forward and reverse primers. A universal TaqMan probe is used and the fluorescence is measured [13]. Another method was developed, which involves RT with a miRNA-specific RT primer, hybridization of the cDNA to a bi-directional extension sequence, and PCR amplification carried out with a miRNA-specific forward primer, a universal reverse primer and SYBR Green [29]. The two-tailed RT-qPCR method uses a hairpin RT primer that binds to both extremes of the mature miRNA; PCR amplification is carried out with miRNA-specific forward and reverse primers and the fluorescence from SYBR Green is quantified [30]. Another approach uses an oligonucleotide that serves as a RT primer and that has a binding region for a universal forward PCR primer; PCR is carried out with a miRNAspecific reverse primer, a universal forward primer and SYBR Green [31]. Another method employs a linear-hairpin variable RT primer that leads to a hairpin CDNA; PCR is carried out with miRNA-specific forward and reverse primers and fluorescence from SYBR Green is measured [14].

A number of papers describing detailed experimental protocols for several of these methods are available [32-37].

\section{isomiRS \& ANALYSIS OF EXPRESSION BY qPCR- BASED METHODS}

In very recent years, results from highthroughput sequencing of small RNA have shown that there is large heterogeneity in the miRNA isoforms (isomiRs) found in different tissues and cells [38,39]. IsomiRs are the result of changes in length or sequence of miRNAs (mainly trimming and tailing) and have been categorized into $5^{\prime}$ isomiRs and $3^{\prime}$ isomiRs, with $3^{\prime}$ isomiRs being more common [38-40]. Multiple isomiRs have been shown to have differential functional effects and implicated in several biological mechanisms and diseases [41-44]. 


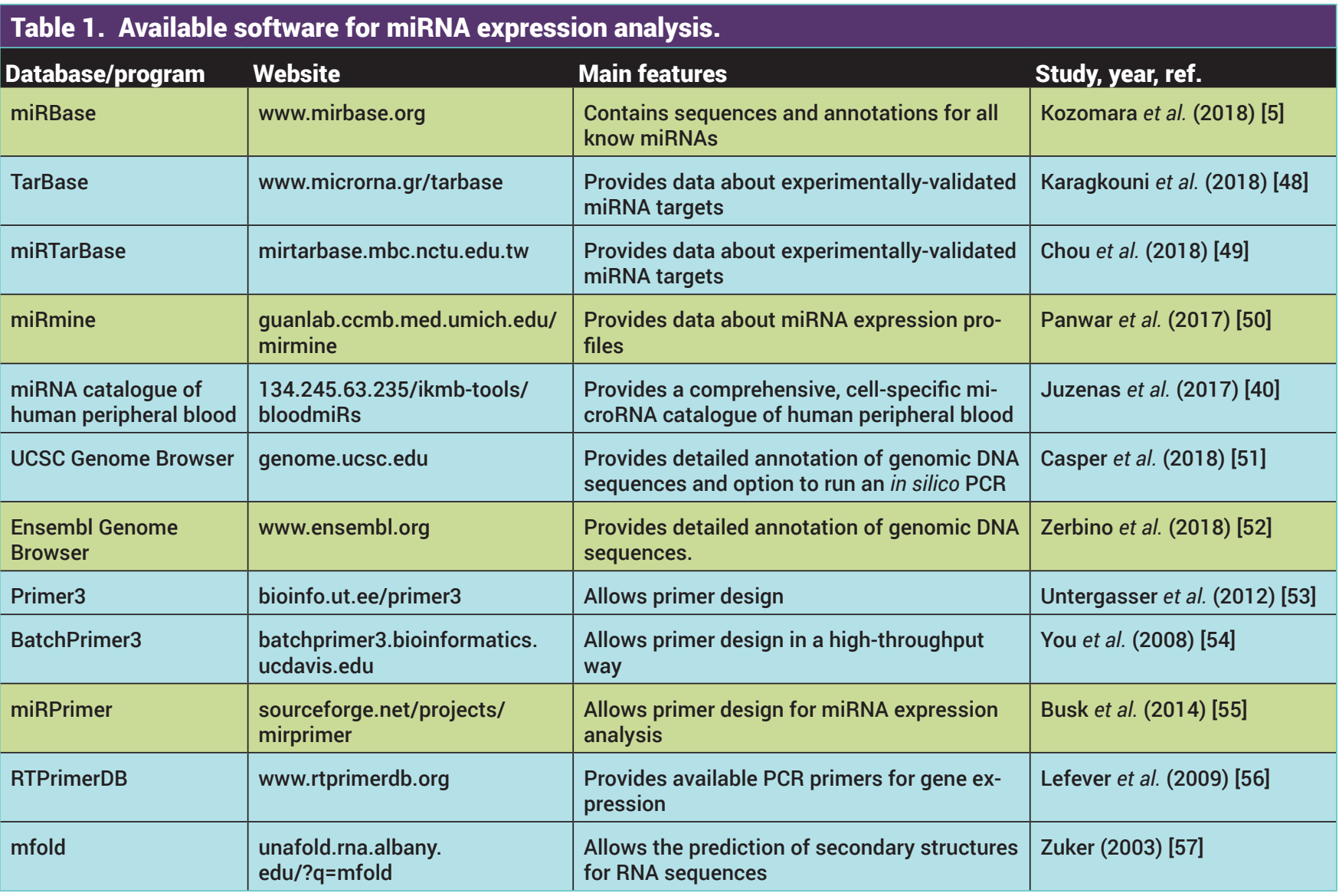

\section{Table 2. Commercially available qPCR assays for miRNA expression analysis.}

\begin{tabular}{|c|c|c|}
\hline Product & Company & Website \\
\hline miRNA Oligo chip & 3D Gene & www.3d-gene.com/en/products/dna/index.html \\
\hline miRNA qPCR assays & Canopy Biosciences & canopybiosciences.com/product-category/nawgen/mirna-qpcr-assays/ \\
\hline microRNA Assays & Eurogentec & secure.eurogentec.com/products/microrna-assays.html \\
\hline $\begin{array}{l}\text { miRCURY"' LNA"' Universal RT } \\
\text { microRNA PCR }\end{array}$ & Exiqon & www.exiqon.com/mirna-pcr \\
\hline All-in-One"' miRNA qRT-PCR & Genecopeia & www.genecopoeia.com/product/qpcr-mirna/ \\
\hline miScript miRNA PCR Arrays & Qiagen & www.qiagen.com/us/shop/pcr/primer-sets/miscript-mirna-pcr-arrays \\
\hline qScript microRNA & Quantabio & www.quantabio.com/products/microrna-profiling \\
\hline SmartChip & Takara & $\begin{array}{l}\text { www.takarabio.com/products/automation-systems/smartchip-real-time-pcr- } \\
\text { system-chips-and-reagents/smartchip-real-time-pcr-system-chips-and-plates }\end{array}$ \\
\hline TaqMan miRNA Assays & Thermo Fisher & $\begin{array}{l}\text { www.thermofisher.com/co/en/home/life-science/pcr/real-time-pcr/real-time- } \\
\text { pcr-assays/mirna-ncrna-taqman-assays/single-tube-mirna-taqman-assays. } \\
\text { html }\end{array}$ \\
\hline $\begin{array}{l}\text { TaqMan Array microRNA } \\
\text { 384-well Cards }\end{array}$ & Thermo Fisher & $\begin{array}{l}\text { www.thermofisher.com/co/en/home/life-science/pcr/real-time-pcr/real-time- } \\
\text { pcr-assays/mirna-ncrna-taqman-assays/taqman-microrna-profiling-using- } \\
\text { 384-well-array-cards.html }\end{array}$ \\
\hline
\end{tabular}


- Recently, it has been shown that the use of assays designed for the analysis of canonical miRNAs can lead to problems due to the finding that isomiRs are quite common and their levels are affected in biological models and diseases [42,45]. In this context, the polyadenylation-based methods are less affected by changes in isomiRs $[45,46]$.

It has been shown that different miRNAspecific forward primers allow the specific identification of mature miRNA isomers with the polyadenylation method [46]. A comparison of two methods (LNA miRCuRY, Exiqon and Taqman, Thermo Fisher) for the analysis of different isomiRs found that both platforms presented a low specificity for the identification of isomiRs [47].

\section{AVAILABLE IN SILICO TOOLS FOR mIRNA EXPRESSION ANALYSIS}

As commercially available qPCR-based assays for miRNA expression are focused mainly on the analysis of canonical miRNAs [45], it is important that lab-wet researchers have the options and computational tools for designing their own assays. There are several freely available programs that are helpful for several steps in the analysis of miRNA expression, including miRbase [5], TarBase [48], a cell-specific microRNA catalogue of human peripheral blood [40], miRTarBase [49], miRmine [50], UCSC Genome Browser [51], Ensembl Genome Browser [52], Primer3 [53], BatchPrimer3 [54], miRPrimer [55], RTPrimerDB [56] and mfold [57] (Table 1).

Busk [55] developed the miRprimer program, which was written in Ruby and is freely available online. This software designs two PCR primers based on the approach created by Balcells et al. (addition of a poly-T tail) [21]. It is an executable file than runs in the DOS environment and uses a .txt file with the sequences of the miRNAs as input.

\section{COMMERCIALLY AVAILABLE METHODS FOR EXPRESSION ANALYSIS OF mIRNAS BASED ON QPCR}

There are a number of commercially available assays for the expression analysis of miRNAs, based on qPCR (Table 2). Some of these existing platforms from several companies in the USA, Europe and Asia are focused on the analysis of single candidates or groups of

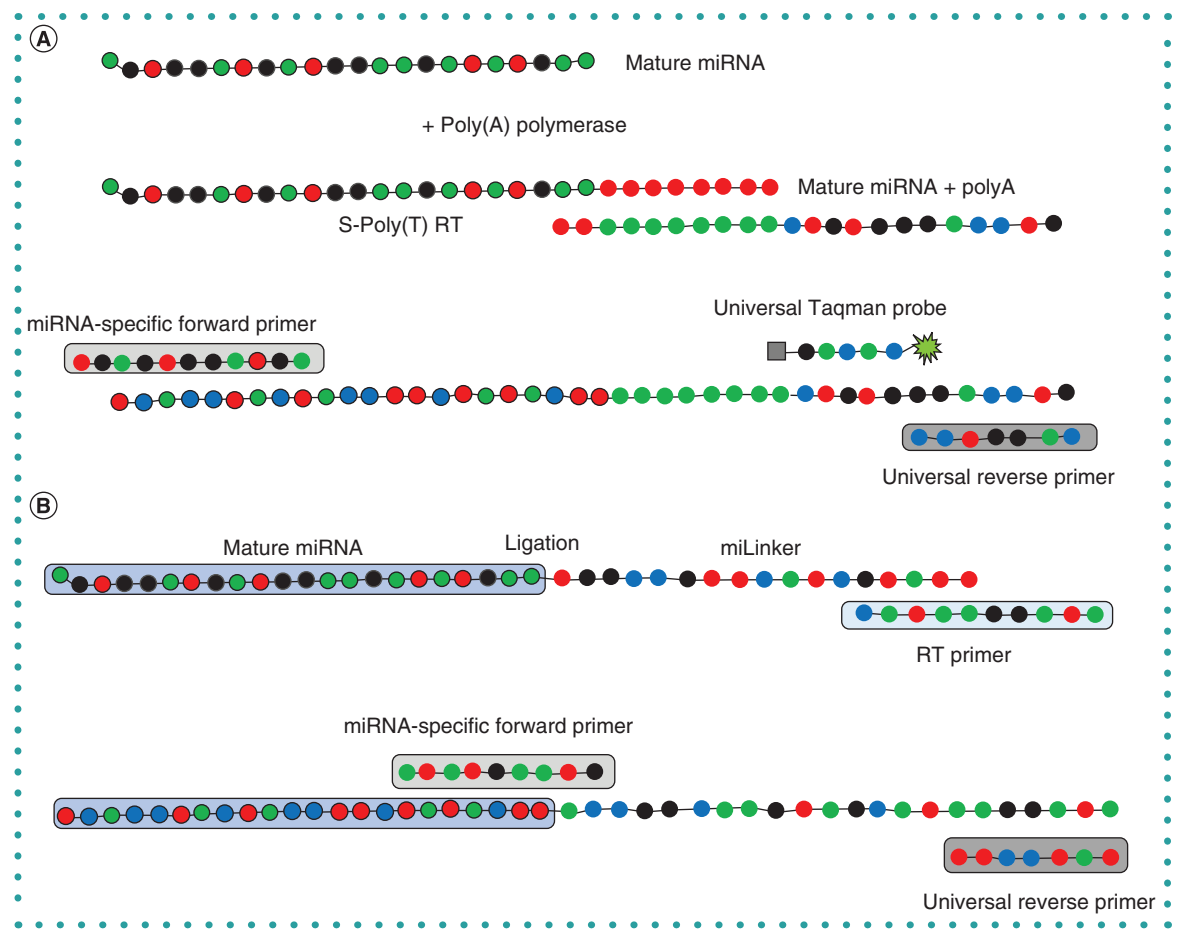

Figure 4. Overview of the qPCR methods developed by (A) Kang et al. [22] and (B) Benes et al. [23]. The approach developed by Kang et al. uses polyadenylation, a polyT adapter and an universal Taqman probe and the method created by Benes et al. involves the ligation of a oligonucleotide, an universal reverse primer and SYBR Green.

RT: Reverse transcriptase.

multiple miRNAs, using different methods for RT and/or PCR amplification and approaches for their quantification (fluorescent probes or DNA-binding dyes) (Table 2). The qPCR-based assays from 3D Gene and Thermo Fisher use an approach based on stem-loop RT primers, and assays from Exiqon, Genecopeia, Qiagen and Quantabio use approaches based on polyadenylation of mature miRNAs. Usually, commercially available qPCR-based assays are designed for the canonical miRNAs defined in the miRBase database [45]. It is possible that the selection of experimental approaches for the selection of these commercially available assays has depended on the advantages of some methods, in addition to issues related to intellectual property from each company (patents). Some of these commercial assays have been available for several years and have been used by a significant number of experimental articles.

\section{OVERVIEW OF METHODS FOR} HIGH-THROUGHPUT mIRNA EXPRESSION ANALYSIS

In recent years, several approaches based on microarrays or NGS have been developed for high-throughput miRNA expression analysis [58,59]. Commonly, these highthroughput methods are used in combination with qPCR-based approaches for the analyses of expression levels of miRNAs [60].

One of the first microarrays for miRNA analyses used 40-mer oligonucleotides printed on slides, with a measurement of fluorescence (transcripts were labeled with biotin and detected with a streptavidin-Alexa647 conjugate) [61,62]. Another method also used oligonucleotides spotted on slides but a dual-channel approach for quantification was employed (RNA was labeled with Сy3 and an oligonucleotide reference set was labeled with Cy5) [63]. The RNA-primed, array-based Klenow enzyme assay was developed, which uses oligonucleotide probes printed onto a slide, with the posterior incorporation of labeled dATPs [64]. The direct miRNA platform was based on the binding of two fluorescent locked nucleic acid (LNA)-DNA oligonucleotide probes to the miRNAs of interest, allowing the analysis of multiple miRNAs and their quantification with a single moleculedetection apparatus [65]. The PanelChip ${ }^{\text {TM }}$ Analysis System was developed and can contain up to 2500 assays, being based 
> on the use of nanowells and qPCR [66]. In recent years, approaches based on RNA sequencing (RNA-seq), which use methods for massive sequencing (such as the ones from Illumina or SOLiD), have shown their large potential for identifying the complete profiles of miRNA variability in several cells and tissues $[40,44,60,67]$.

A study compared the results from six microarray platforms (Agilent, Ambion, Combimatrix, Exiqon, Illumina and Invitrogen), of which three (Exiqon, Ambion, and Agilent) showed greater reproducibility and a high rate of true calls for differentially expressed (DE) miRNAs. [68]. An analysis of the differences between one microarray (Illumina) and two NGS (SOLiD and Illumina) platforms and the NanoString nCounter system showed a good correlation between them and a better performance of NGS, including analyses of formalinfixed, paraffin-embedded tissues [69].

\section{COMPARISONS OF AVAILABLE METHODS FOR EXPRESSION ANALYSIS OF mIRNAS BASED ON qPCR}

A comparison of the results from a qPCR-array (TaqMan Rodent MicroRNA Arrays, Applied Biosystems) and a microarray platform (LC Sciences) found high reproducibility for data generated by the qPCR array and a low correlation $(r=-0.443)$ between the two platforms, particularly for miRNAs with low expression [70]. A study reported the comparison of the results generated from the microarray platforms (Affymetrix, Illumina and Agilent), ultra-high-throughput sequencing (Illumina platform) and qPCR (TaqMan Human MicroRNA A Array v2.0 from Applied Biosystems) for miRNA levels [71]. It was found that the results from qPCR were similar to those from NGS and the Agilent microarray [71].

An analysis of the results from two qPCRbased platforms (miRCURY Ready-to-Use PCR, Exiqon, and TaqMan Human MicroRNA Array v3.0, Life Technologies) with an arraybased system (GeneChip miRNA 2.0 Array, Affymetrix) found that the array-based platform has a lower sensitivity and that the miRCURY system has a better sensitivity than the TaqMan platform [72].

An analysis of the differences in the results from Taqman miRNA assays (Life Technologies) and miRCURY LNA Universal RT microRNA PCR assays (Exiqon) found an effect of small RNA enrichment on the expression levels of miRNAs with these methods and that the variability was lower with the TaqMan platform [73]. Mou et al. compared three methods: stem-loop qPCR, poly $(A)$ tailing and miQPCR, and found that the poly(A)-based method has a better ability to detect miRNAs with low expression levels [74].

A collaborative team compared the results from 12 platforms (from nine companies) for 16 mandatory and four optional samples, for 196 miRNAs. Seven of these platforms were based on qPCR: miRCury (Exiqon), OpenArray (Life Technologies), TaqMan Cards (Life Technologies), TaqMan Cards preAmp (Life Technologies), miScript (Qiagen), qScript (Quanta BioSciences) and SmartChip (WaferGen) [75]. Three other platforms tested were based on microarrays and two on NGS. They found that the miRCury system showed the highest specificity; SmartChip and miRCury showed the highest reproducibility; miScript and qScript showed the highest accuracy; and that SmartChip and miScript showed the highest sensitivity. The concordance for the identification of differentially expressed miRNAs between platforms was low, with only $3 \%$ of the miRNAs found as DE by all platforms [75].

Absolute quantification using the droplet digital PCR (ddPCR) system actually outperforms conventional RT-qPCR analysis with standard curves (using miRNA mimics), especially for low-expressed miRNAs, and in addition to this, it does not rely on reference genes for normalization [76,77].

\section{NORMALIZATION, QUALITY CONTROL \& REPORTING}

A study using a microarray platform to analyze miRNAs in 13 human tissues found that several miRNAs had a higher stability miR-191, miR-93, miR-106a, miR-17-5p and miR-25 - with these being higher than the one found for the U6 snoRNA [78]. The use of external controls (spike-in miRNAs) is important for normalization [73]; spike-in controls are commonly used, mostly to account for variability in RNA extractions (especially from fluids in which low miRNA levels are detected) rather than to employ them for relative quantifications. In plants, several miRNAs have been proposed as reference genes for normalization, such as miR-156b and miR-1520d in soybean, and miR-172,
miR-168 and miR-390 in cotton $[79,80]$.

Other articles have proposed and reviewed different approaches for normalization of miRNA expression data [81-85], particularly for platforms designed for the multiplex analysis of candidate miRNAs. It is possible to carry out a normalization of miRNA expression levels based on geometric means for multiple reference genes, as it is done for analysis of mRNAs [86].

In 2009, a group of international researchers published the Minimum Information for Publication of Quantitative Real-Time PCR Experiments (MIQE) Guidelines [87], which are useful for authors and reviewers. It includes points about multiple important aspects of qPCR-based studies, including experimental design, samples, nucleic acid extraction, RT, qPCR target information, qPCR oligonucleotides, qPCR protocol, qPCR validation and data analysis [87]. One of the key elements is the need for reporting the sequences of the PCR primers used.

The importance of the use of negative controls for $\mathrm{RT}$ and $\mathrm{qPCR}$ reactions (nontemplate controls for RT and PCR) to identify the specificity of the reactions has been highlighted [73]. It has been shown that the results of the RT depended on the characteristics of the RT enzyme, samples, RNA concentrations and assays [88].

\section{FUTURE PERSPECTIVE: CONSIDERATIONS FOR FUTURE QPCR-BASED METHODS FOR EXPRESSION ANALYSIS OF MIRNAS}

Several aspects are important to consider in future publications and applications related to the qPCR-based methods for the analysis of expression of miRNAs: as mentioned above, a large fraction of the available methods is focused on the analysis of mature miRNA levels, highlighting the need for the implementation of additional approaches for the quantitative study of expression levels of pri- and pre-miRNAs [10].

Development and refinement of methods that are focused on the implementation of multiplex assays for quantification of miRNAs expression would facilitate high-throughput analyses, particularly with methods that are cost effective, including monochrome approaches that use a single fluorescent dye [89]. As discussed above, there is only one freely available program [55] for the specific 
design of primers for miRNA expression analysis. It highlights the important need for the development of additional freely available programs for design of primers for different methods for miRNA expression analysis, particularly in the context of the challenge of studying isomiRs that are not currently targeted by commercially available methods.

As the MIQE guidelines [87] were developed for gene expression experiments in general, an adjusted MIQE guideline considering the particularities of the analysis of miRNAs expression would facilitate the reporting and reproducibility of those type of experiments. There is a need for more experimental studies exploring the best miRNAs for normalization in expression studies [78], incorporating a larger number of tissues and cell types. Additional comparisons of commercially available assays [75] for expression analysis of miRNAs would be helpful, considering that novel commercial platforms have been developed in recent years.

\section{AUTHOR CONTRIBUTIONS}

DAF conceived the idea, reviewed the literature, contributed to the generation of tables and figures and wrote and revised the manuscript: YG-G conceived the idea, reviewed the literature, contributed to the generation of tables and figures and wrote and revised the manuscript; LJC-V reviewed the literature and contributed to the writing and revision of the manuscript; GEB reviewed the literature and contributed to the writing and revision of the manuscript.

\section{FINANCIAL \& COMPETING INTERESTS DISCLOSURE}

YG-G is supported by a PhD fellowship from Centro de Estudios Interdisciplinarios Básicos y Aplicados CEIBA (Rodolfo Llinás Program). DAF is supported by research grants from Colciencias and VCTI. The authors have no other relevant affiliations or financial involvement with any organization or entity with a financial interest in or financial conflict with the subject matter or materials discussed in the manuscript apart from those disclosed.

No writing assistance was utilized in the production of this manuscript.

\section{OPEN ACCESS}

This work is licensed under the AttributionNonCommercial-NoDerivatives 4.0 Unported License. To view a copy of this license, visit http://creativecommons.org/licenses/ by-nc-nd/4.0/

\section{REFERENCES}

1. Gulyaeva LF, Kushlinskiy NE. Regulatory mechanisms of microRNA expression. J. Translat. Med. 14(1), 143 (2016)

2. Paul $P$, Chakraborty $A$, Sarkar $D$ et al. Interplay between miRNAs and human diseases. J. Cell. Physiol. 233(3), 2007-2018 (2018).

3. Zhang $Y$, Yun $Z$, Gong $L$ et al. Comparison of miRNA evolution and function in plants and animals. MicroRNA 7(1) 4-10 (2018).

4. Wang J, Mei J, Ren G. Plant microRNAs: biogenesis homeostasis, and degradation. Front. Plant Sci. 10, 360 (2019).

5. Kozomara A, Birgaoanu M, Griffiths-Jones S. miRBase from microRNA sequences to function. Nucl. Acids Res. 47(D1), D155-D162 (2018).

6. Kong W, Zhao JJ, He L, Cheng JQ. Strategies for profiling microRNA expression. J. Cell. Physiol. 218(1), 22-25 (2009).

7. Pritchard CC, Cheng HH, Tewari M. MicroRNA profiling: approaches and considerations. Nat. Rev. Genet. 13(5), 358-369 (2012).

8. Jacobsen N, Andreasen D, Mouritzen P. Profiling microRNAs by real-time PCR. Method. Mol. Biol. 732, 39-54 (2011).

9. Falagas ME, Pitsouni El, Malietzis GA, Pappas G. Comparison of PubMed, Scopus, Web of Science, and Google Scholar. strengths and weaknesses. FASEB J. 22(2), 338-342 (2008)

10. Schmittgen TD, Jiang J, Liu Q, Yang L. A high-throughput method to monitor the expression of microRNA precursors. Nucl. Acids Res. 32(4), e43 (2004).

11. Chen C, Ridzon DA, Broomer AJ et al. Real-time quantification of microRNAs by stem-loop RT-PCR. Nucl. Acids Res. 33(20), e179 (2005).

12. Shi R, Chiang VL. Facile means for quantifying microRNA expression by real-time PCR. BioTechniques 39(4), 519-525 (2005).

13. Huang T, Yang J, Liu $\mathrm{G}$ et al. Quantification of mature microRNAs using pincer probes and real-time PCR amplification. PLOS One 10(3) e0120160 (2015).

14. Lan L, Guo Q, Nie H et al. Linear-hairpin variable primer RT-qPCR for microRNA. Chem. Sci. 10(7), 2034-2043 (2019)

15. Ponchel F, Toomes $\mathrm{C}$, Bransfield $\mathrm{K}$ et al. Real-time PCR based on SYBR-Green I fluorescence: an alternative to the TaqMan assay for a relative quantification of gene rearrangements, gene amplifications and micro gene deletions. BMC Biotechnol 3, 18 (2003).

16. Wang Y, Zhou J, Chen Y et al. Quantification of distinct let-7 microRNA family members by a modified stem-loop RT-qPCR. Mol. Med. Report. 17(3), 3690-3696 (2018).

17. Lao K, Xu NL, Yeung V, Chen C, Livak KJ, Straus NA. Multiplexing RT-PCR for the detection of multiple miRNA species in small samples. Biochem. Biophys. Res. Comm 343(1) 85-89 (2006).

18. Jung $U$, Jiang $X$, Kaufmann SH, Patzel V. A universal TaqMan-based RT-PCR protocol for cost-efficient detection of small noncoding RNA. RNA 19(12), 1864-1873 (2013).
19. Tong L, Xue H, Xiong L, Xiao J, Zhou Y. Improved RT-PCR assay to quantitate the pri-, pre--, and mature microRNAs with higher efficiency and accuracy. Mol. Biotechnol. 57(10), 939-946 (2015).

20. Ro S, Park C, Jin J, Sanders KM, Yan W. A PCR-based method for detection and quantification of small RNAs. Biochem. Biophys. Res. Comm. 351(3), 756-763 (2006).

21. Balcells I, Cirera S, Busk PK. Specific and sensitive quantitative RT-PCR of miRNAs with DNA primers. BMC Biotechnol. 11, 70 (2011)

22. Kang $\mathrm{K}$, Zhang $\mathrm{X}$, Liu $\mathrm{H}$ et al. A novel real-time PCR assay of microRNAs using S-Poly(T), a specific oligo(dT) reverse transcription primer with excellent sensitivity and specificity. PLoS One 7(11), e48536 (2012).

23. Benes $\mathrm{V}, \mathrm{Collier} \mathrm{P}$, Kordes $\mathrm{C}$ et al. Identification of cytokine-induced modulation of microRNA expression and secretion as measured by a novel microRNA specific qPCR assay. Sci. Rep. 5, 11590 (2015).

24. Kumar P, Johnston BH, Kazakov SA. miR-ID: a novel, circularization-based platform for detection of microRNAs. RNA 17(2), 365-380 (2011)

25. Ge Q, Tian F, Zhou $Y$ et al. A universal linker-RT PCR based quantitative method for the detection of circulating miRNAs. Analyt. Method. 6(22), 9101-9107 (2014).

26. Honda S, Kirino Y. Dumbbell-PCR: a method to quantify specific small RNA variants with a single nucleotide resolution at terminal sequences. Nucl. Acids Res. 43(12), e77 (2015).

27. Zheng W, Di Y, Liu Y et al. Development and application of a novel reverse transcription real-time PCR method for miR-499 quantification. Clin. Biochem. 46(15), 1566-1571 (2013).

28. Li X, Ni M, Zhang C, Ma W, Zhang Y. A convenient system for highly specific and sensitive detection of miRNA expression. RNA 20(2), 252-259 (2014).

29. Kim KJ, Kwak J, Lee JH, Lee SS. Real-time qRT-PCR assay for the detection of miRNAs using bi-directional extension sequences. Analyt. Biochem. 536, 32-35 (2017)

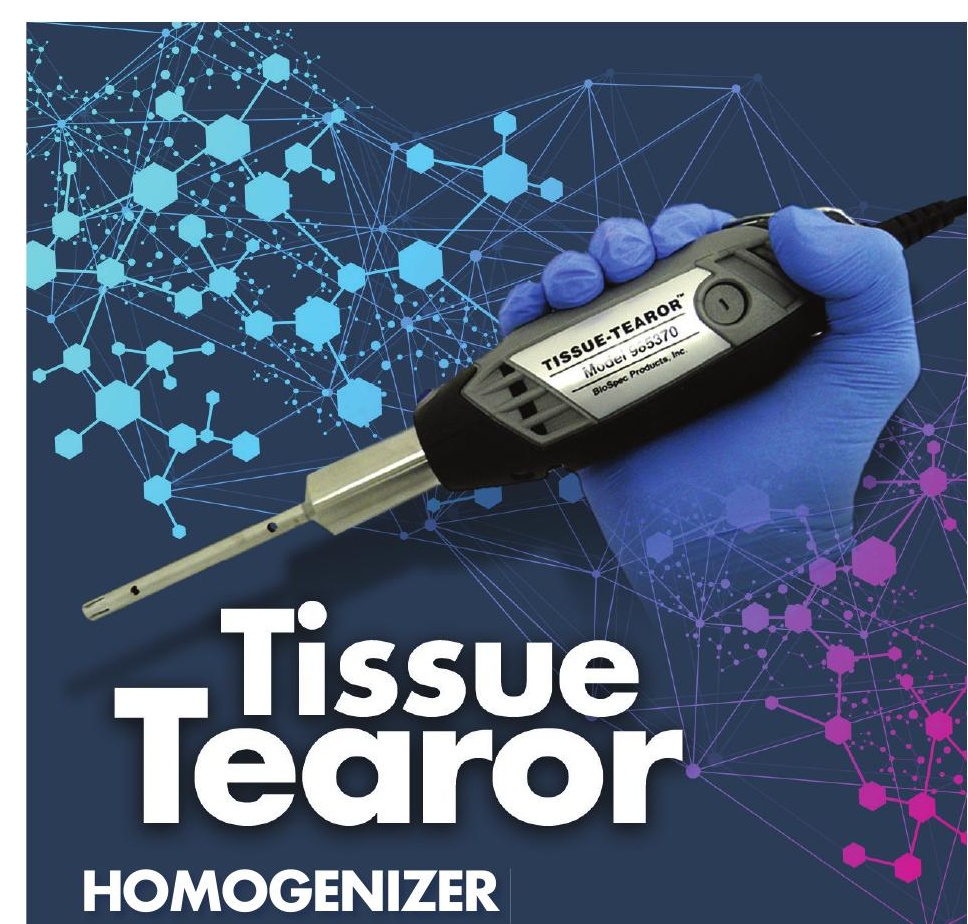

- Cell Lysis in Seconds

- No Sample Heating

- Choice of Four Probe Sizes

BioSpec Products, Inc.

$\mathrm{PO}$ Box 788

Bartlesville, OK 74005, USA

800-617-3363 $\left.8_{8}^{8}\right)_{0}^{\circ}$

BIOSPECPRODUCTS 
30. Androvic P, Valihrach L, Elling J, Sjoback R, Kubista M. Two-tailed RT-qPCR: a novel method for highly accurate miRNA quantification. Nucl. Acids Res. 45(15), e144 (2017).

31. Zhang W, Zhang J, Zhang Q, Hu F, Gu Y. Highly specific real-time quantification of diverse microRNAs in human samples using universal primer set frame. Analyt. Biochem. 543, 71-78 (2018).

32. Leti F, Distefano JK. miRNA quantification method usin quantitative polymerase chain reaction in conjunction with C q method. Method. Mol. Biol. 1706, 257-265 (2018).

33. Chugh P, Tamburro K, Dittmer DP. Profiling of pre-micro RNAs and microRNAs using quantitative real-time PCR (qPCR) arrays. J. Vis. Exp. 46, pii: 2210 (2010).

34. Zeka F, Mestdagh P, Vandesompele J. RT-qPCR-based quantification of small Non-Coding RNAs. Method. Mol. Biol. 1296, 85-102 (2015).

35. Zollner H, Hahn SA, Maghnouj A. Quantitative RT-PCR specific for precursor and mature miRNAs. Method. Mol. Biol. 1095, 121-134 (2014).

36. Kramer MF. Stem-loop RT-qPCR for miRNAs. Current Protocols in Molecular Biology (Chapter 15). Unit 15, 10 (2011)

37. Salone V, Rederstorff M. Stem-loop RT-PCR based quantification of small Non-Coding RNAs. Method. Mol. Biol. 1296, 103-108 (2015)

38. Guo $L$, Chen $F$. A challenge for miRNA: multiple isomiRs in miRNAomics. Gene 544(1), 1-7 (2014).

39. Bofill-De Ros X, Yang A, Gu S. IsomiRs: expanding the miRNA repression toolbox beyond the seed. Biochim. Biophys. Acta Gene Regul. Mech. pii: S1874-9399(18)30539-X (2019)

40. Juzenas $\mathrm{S}$, Venkatesh $\mathrm{G}$, Hubenthal $\mathrm{M}$ et al. A compre hensive, cell specific microRNA catalogue of human peripheral blood. Nucl. Acids Res. 45(16), 9290-9301 (2017).

41. Telonis AG, Magee R, Loher P, Chervoneva I, Londin $E$, Rigoutsos I. Knowledge about the presence or absence of miRNA isoforms (isomiRs) can successfully discriminate amongst 32 TCGA cancer types. Nucl. Acids Res. 45(6), 2973-2985 (2017).

42. Nejad C, Pillman KA, Siddle KJ et al. miR-222 isoforms are differentially regulated by type-I interferon RNA 24(3), 332-341 (2018).

43. Yu F, Pillman KA, Neilsen CT et al. Naturally existing isoforms of miR-222 have distinct functions. Nucl. Acids Res. 45(19), 11371-11385 (2017).

44. Cloonan N, Wani S, Xu Q et al. MicroRNAs and their isomiRs function cooperatively to target common biological pathways. Genome Biol. 12(12), R126 (2011).

45. Pillman KA, Goodall GJ, Bracken CP, Gantier MP. miRNA length variation during macrophage stimulation confounds the interpretation of results: implications for miRNA quantification by RT-qPCR. RNA 25(2), 232-238 (2019).

46. Nejad C, Pepin G, Behlke MA, Gantier MP. Modified polyadenylation-based RT-qPCR increases selectivity of amplification of 3'-MicroRNA isoforms. Front. Genet. 9, 11 (2018)
47. Magee R, Telonis AG, Cherlin T, Rigoutsos I, Londin E. Assessment of isomiR discrimination using commercial qPCR methods. Non-Coding RNA 3(2), (2017).

48. Karagkouni D, Paraskevopoulou MD, Chatzopoulos S et al. DIANA-TarBase v8: a decade-long collection of experimentally supported miRNA-gene interactions. Nucl. Acids Res. 46(D1), D239-D245 (2018).

49. Chou $\mathrm{CH}$, Shrestha $\mathrm{S}$, Yang $\mathrm{CD}$ et al. miRTarBase update 2018: a resource for experimentally validated microRNA-target interactions. Nucl. Acids Res. 46(D1), D296-D302 (2018)

50. Panwar B, Omenn GS, Guan Y. miRmine: a database of human miRNA expression profiles. Bioinformatics $33(10)$ 1554-1560 (2017)

51. Casper J, Zweig AS, Villarreal C et al. The UCSC Genome Browser database: 2018 update. Nucl. Acids Res. 46(D1), D762-D769 (2018)

52. Zerbino DR, Achuthan P, Akanni W et al. Ensembl 2018. Nucl. Acids Res. 46(D1), D754-D761 (2018).

53. Untergasser A, Cutcutache I, Koressaar T et al. Primer3: new capabilities and interfaces. Nucl. Acids Res. 40(15) e115 (2012)

54. You FM, Huo N, Gu YQ et al. BatchPrimer3: a high throughput web application for PCR and sequencing primer design. BMC Bioinformat. 9, 253 (2008).

55. Busk PK. A tool for design of primers for microRNA-specific quantitative RT-qPCR. BMC Bioinformat. 15, 29 (2014)

56. Lefever S, Vandesompele J, Speleman F, Pattyn F. RTPrimerDB: the portal for real-time PCR primers and probes. Nucl. Acids Res. 37(Database issue), D942-D945 (2009).

57. Zuker M. Mfold web server for nucleic acid folding and hybridization prediction. Nucl. Acids Res. 31(13), 3406-3415 (2003).

58. Ouyang T, Liu Z, Han Z, Ge Q. MicroRNA detection specficity. recent advances and future perspective. Analyt. Chem. 91(5), 3179-3186 (2019).

59. Yin JQ, Zhao RC, Morris KV. Profiling microRNA expression with microarrays. Trends Biotechnol. 26(2), 70-76 (2008)

60. Hunt EA, Broyles D, Head T, Deo SK. MicroRNA detection: current technology and research strategies. Annu. Rev. Anal. Chem. (Palo Alto Calif.) 8, 217-237 (2015).

61. Liu CG, Calin GA, Meloon B et al. An oligonucleotide microchip for genome-wide microRNA profiling in human and mouse tissues. Proc. Natl Acad. Sci. USA 101(26), 9740-9744 (2004)

62. Liu CG, Calin GA, Volinia S, Croce CM. MicroRNA expression profiling using microarrays. Nat. Protocol. 3(4), 563-578 (2008).

63. Thomson JM, Parker J, Perou CM, Hammond SM. A custom microarray platform for analysis of microRNA gene expression. Nat. Method. 1(1), 47-53 (2004)

64. Nelson PT, Baldwin DA, Scearce LM, Oberholtzer JC, Tobias JW, Mourelatos Z. Microarray-based, high-throughput gene expression profiling of microRNAs. Nat. Method. 1(2), 155-161 (2004).

65. Neely LA, Patel S, Garver J et al. A single-molecule method for the quantitation of microRNA gene expression. Nat. Method. 3(1), 41-46 (2006).

\section{BioTechniques}

\section{TIME TO RENEW YOUR SUBSCRIPTION}

Subscriptions to BioTechniques need to be renewed every year. Don't miss out on all of the comprehensive reviews, novel research articles, and insightful features found each month in the pages of BioTechniques.
Renew Today and Choose Your

SUBSCRIPTIONS ARE FREE TO QUALIFIED SUBSCRIBERS Preferred Formats
PRINT OR DIGITAL

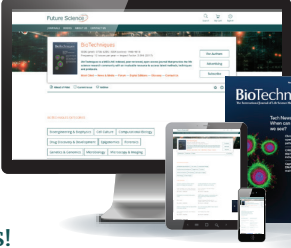

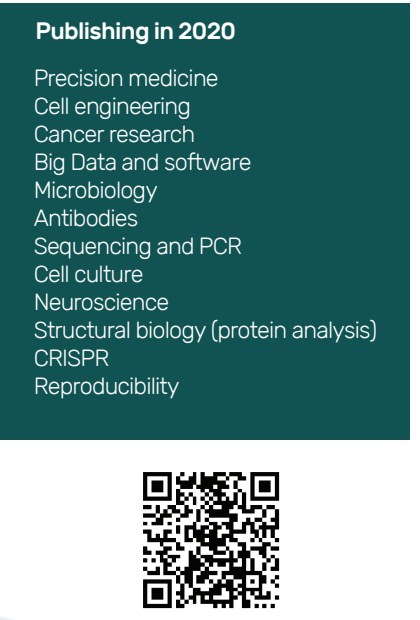

http://bit.ly/BTNrenew
66. Hsieh $\mathrm{CH}$, Chen WM, Hsieh YS et al. A novel multi-gene detection platform for the analysis of miRNA expression. Sci. Rep. 8(1), 10684 (2018).

67. Morin RD, O'connor MD, Griffith $\mathrm{M}$ et al. Application of massively parallel sequencing to microRNA profiling and discovery in human embryonic stem cells. Genom Res. 18(4), 610-621 (2008).

68. Git A, Dvinge $\mathrm{H}$, Salmon-Divon $\mathrm{M}$ et al. Systematic comparison of microarray profiling, real-time PCR, and next-generation sequencing technologies for measuring (2010).

69. Tam S, De Borja R, Tsao MS, Mcpherson JD. Robust global microRNA expression profiling using next-generation sequencing technologies. Lab. Invest. 94(3), 350-358 (2014)

70. Chen Y, Gelfond JA, Mcmanus LM, Shireman PK Reproducibility of quantitative RT-PCR array in miRNA expression profiling and comparison with microarray analysis. BMC Genomics 10, 407 (2009).

71. Pradervand S, Weber J, Lemoine F et al. Concordance among digital gene expression, microarrays, and qPCR when measuring differential expression of microRNAs. BioTechniques 48(3), 219-222 (2010)

72. Jensen SG, Lamy P, Rasmussen MH et al. Evaluation of two commercial global miRNA expression profiling platforms for detection of less abundant miRNAs. BMC Genomics 12, 435 (2011).

73. Redshaw N, Wilkes T, Whale A, Cowen S, Huggett J, Foy CA. A comparison of miRNA isolation and RT-qPCR technologies and their effects on quantification accuracy and nologies and their effects on quantification accuracy
repeatability. BioTechniques 54(3), 155-164 (2013).

74. Mou G, Wang K, Xu D, Zhou G. Evaluation of three RTqPCR-based miRNA detection methods using seven rice miRNAs. Biosci. Biotechnol. Biochem. 77(6), 1349-1353 (2013).

75. Mestdagh P, Hartmann N, Baeriswyl L et al. Evaluation of quantitative miRNA expression platforms in the microRNA quality control (miRQC) study. Nat. Method. 11(8), 809-815 (2014).

76. Hindson CM, Chevillet JR, Briggs $\mathrm{HA}$ et al. Absolute quantification by droplet digital PCR versus analog real-time PCR. Nat. Method. 10(10), 1003-1005 (2013).

77. Huggett JF, Cowen S, Foy CA. Considerations for digital PCR as an accurate molecular diagnostic tool. Clin. Chem. 61(1), 79-88 (2015).

78. Peltier HJ, Latham GJ. Normalization of microRNA expression levels in quantitative RT-PCR assays: identification of suitable reference RNA targets in normal and cancerous human solid tissues. RNA 14(5), 844-852 (2008).

79. Kulcheski FR, Marcelino-Guimaraes FC, Nepomuceno AL Abdelnoor RV, Margis R. The use of microRNAs as reference genes for quantitative polymerase chain reaction in soybean. Analyt. Biochem. 406(2), 185-192 (2010).

80. Fausto AKS, Silva TDF, Romanel E, Vaslin MFS. microRNAs as reference genes for quantitative PCR in cotton. PLoS One 12(4), e0174722 (2017).

81. Wylie D, Shelton J, Choudhary A, Adai AT. A novel mean-centering method for normalizing microRNA expression from high-throughput RT-qPCR data. BMC Res. Notes 4, 555 (2011).

82. Mohammadian A, Mowla SJ, Elahi E, Tavallaei M, Nourani MR, Liang Y. Normalization of miRNA qPCR high-throughput data: a comparison of methods. Biotechnol. Lett. 35(6), 843-851 (2013).

83. Qureshi R, Sacan A. A novel method for the normalization of microRNA RT-PCR data. BMC Med. Genom. 6(Suppl. 1), S14 (2013).

84. Mestdagh P, Van Vlierberghe P, De Weer A et al. A nove and universal method for microRNA RT-qPCR data normalization. Genome Biol. 10(6), R64 (2009).

85. Schwarzenbach H, Da Silva AM, Calin G, Pantel K. Data normalization strategies for MicroRNA quantification. Clin. Chem. 61(11), 1333-1342 (2015)

86. Vandesompele J, De Preter K, Pattyn F et al. Accurate normalization of real-time quantitative RT-PCR data by geometric averaging of multiple internal control genes. Genome Biol. 3(7), RESEARCH0034 (2002).

87. Bustin SA, Benes V, Garson JA et al. The MIQE guidelines: minimum information for publication of quantitative real-time PCR experiments. Clin. Chem. 55(4), 611-622 (2009).

88. Bustin S, Dhillon HS, Kirvell S et al. Variability of the reverse transcription step: practical implications. Clin. Chem. 61(1), 202-212 (2015)

89. Cawthon RM. Telomere length measurement by a novel monochrome multiplex quantitative PCR method. NuCl. Acids Res. 37(3), e21 (2009). 